\section{MS9-P15 Polymorphism and phase stability of ketoprofen salts}

M. Ben Nasr ${ }^{1}$, A. Doudouh ${ }^{1}$, P. Durand ${ }^{1}$, A. Gansmuller ${ }^{1}$, E. Aubert $^{1}$, E. Espinosa ${ }^{1}$

\section{Laboratoire CRM2 Université de Lorraine}

email: mahjouba.ben-nasr@univ-lorraine.fr

Ketoprofen is a Non-Steroidal Anti-Inflammatory Drug (NSAID) of propionic acid class having analgesic and antipyretic effects [1]. It exhibits poor water solubility and dissolution rate [2]. To enhance its solubility, ketoprofen has been formulated and marketed as a trometamol salt [3].

Up to now the structures of ketoprofen salts were unknown. Recently, we have succeeded to obtain single crystals of a salt of racemic ketoprofen with trometamol, as well as two polymorphs of S-ketoprofen-trometamol salts (Fig.1). The prepared salts were characterized by using single-crystal and powder X-ray diffraction, DSC, FT-IR and 13C CP-MAS NMR, coupled with Density Functional Theory calculations. The structures and properties of these salts, which show enhanced aqueous solubility as compared to pure ketoprofen, will be discussed.

References:

[1] M., Dixit, P., Kulkarni, R., Vaghela, Trop. J. Pharm. Res. 12, 317-322 (2013).

[2] P. S. Yadav, V. Kumar, U. P. Singh, H. R. Bhat, B. 1. Mazumder, Saudi Pharm. J. 21, 77- 84 (2013).

[3] B. J. Sweetman, Acute Pain, 4, 109-115 (2003).
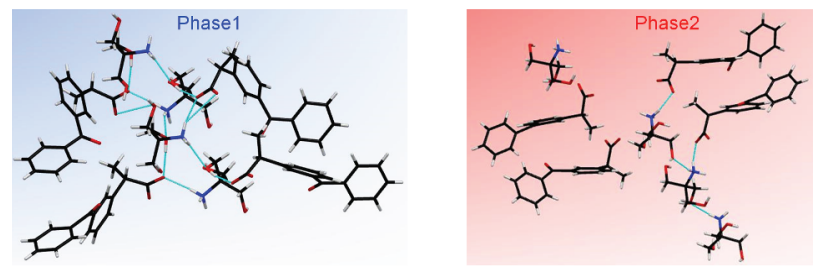

Figure 1. Crystal structures of two polymorphs of S-ketoprofen-trometamol salts. N-H...O and O-H...O hydrogen bonds are shown as blue lines

Keywords: Crystallization, ketoprofen, trometamol, salt, hydrogen bonding, solubility.

\section{MS9-P16 Conserved hydrogen bonding in} tetrahydrocarbazolone derivatives: Influence of solution-state assembly on crystal form nucleation

Katharina Fucke ${ }^{1}$, Robert M. Edkins ${ }^{2,3}$, Elliott Hayden ${ }^{1}$

1. School of Medicine, Pharmacy and Health, Durham University Queen's Campus, University Boulevard, Stockton-on-Tees, TS17 6BH, United Kingdom

2. Institut fuer Anorganische Chemie, Julius-Maximilians-Universitaet Wuerzburg, Am Hubland, 97074 Wuerzburg, Germany

3. Department of Chemistry, Durham University, South Road, Durham, DH1 3LE, United Kingdom

email: katharina.fucke@durham.ac.uk

Different crystal forms (polymorphs) may vary substantially in their physico-chemical characteristics, including melting point, chemical and physical stability, solubility and dissolution rate, the latter of which represents both a challenge and an opportunity for the pharmaceutical industry. ${ }^{1}$ Bioactive molecules typically have multiple functional groups, enabling them to interact with receptors and thus show pharmacological action. Furthermore, as drug molecules become ever larger, they tend to show increased flexibility. These two factors make investigations and predictions of the crystallisation behaviour of most drug molecules inherently difficult. ${ }^{2}$ In this study, we investigate two tetrahydrocarbazolone derivatives, as they represent core fragments of many antibacterial and antiviral drugs and prodrugs, ${ }^{3}$ whilst having a rigid core with only one hydrogen-bond (HB) donor and one $\mathrm{HB}$ acceptor functionality, enabling us to deconvolute the influence of specific functional groups. In addition, the influence of the position of methylation on the existence of supramolecular synthons is probed. Ortho-methylated tetrahydrocarbazolone (OCB) can exist in four polymorphs, three of which show the anticipated dimer formation, a synthon proved to exist in the pre-crystallisation solution. The thermodynamically stable polymorph, however, crystallises in a catemer motif but has a considerably longer nucleation time. When moving the methyl group from the ortho- to the para-position (PCB), only dimer formation was observed, while the different polymorphs become very close in energy and concomitant crystallisation occurred. Thus, subtle changes in molecular structure can have profound influences on crystallisation behaviour. It is also predicted that a bio-isosteric replacement of the $\mathrm{CH}_{3}$ group of $\mathrm{OCB}$ with $\mathrm{CF}_{3}$ will further stabilise the catemer, highlighting a potential problem for the design and subsequent formulation of new drugs.

${ }^{1}$ D. J. W. Grant, in Polymorphism in Pharmaceutical Solids, ed. H. G. Brittain, Marcel Dekker Inc., New York, 1999, pp. 1-33.

2 S. L. Price, Chem. Soc. Rev., 2014, 43, 2098.

3 (a) X. Li and R. Vince, Bioorg. Med. Chem., 2006, 14, 2942; (b) G. Periyasami, R. Raghunathan, G. Surendiran and N. Mathivanan, Bioorg. Med. Chem. Lett., 2008, 18, 2342; (c) K. S. Gudmundsson, P. R. Sebahar, L. D. A. Richardson, J. G. Catalano, S. D. Boggs, A. Spaltenstein, P. B. Sethna, K. W. Brown, R. Harvey and K. R. Romines, Bioorg. Med. Chem. Lett., 2009, 19, 3489. 